\title{
An atypical pituitary adenoma with a high degree of malignancy: a case report
}

\author{
Songquan Wang ${ }^{1,2+}$, Deling $\mathrm{Li}^{1,2+}$, Guijun $\mathrm{Jia}^{1,2^{*}}$ and Ming $\mathrm{Ni}^{1,2}$
}

\begin{abstract}
Background: Pituitary carcinoma is very rare and hard to diagnose. However, some atypical pituitary adenomas present with a high rate of proliferation and extensive invasion, suggesting a tumor with a high degree of malignancy.

Case presentation: Here, we present a case study of this type of tumor. A 46 years old women was admitted for headaches and impaired vision, and MRI and CT revealed a sellar lesion. She underwent a transsphenoidal surgery followed by radiotherapy, but the tumor recurred only one month later. She decided to undergo a second and third operation. The final pathology showed a non-functioning adenoma that was approximately $80 \%$ ki-67-positive and extensively p53-positive. No metastasis was found. The tumor progressed extremely quickly, and the patient died 7 months after the initial diagnosis.
\end{abstract}

Conclusions: A pituitary adenoma like this should be treated like a carcinoma, and early treatment may increase the likelihood of survival. A traditional diagnosis standard is unfit for this type of tumor.

Keywords: Atypical pituitary adenoma, Pituitary carcinoma, Pathology, Invasiveness

\section{Background}

Pituitary adenomas comprise approximately $15 \%$ of all intracranial tumors. These tumors arise from adenohypophyseal cells and were once considered to be the most common benign neoplasm in the sellar region. However, recent evidence has suggested that while metastasis is rare in these tumors, their designation as benign should be revised. Some aggressive tumors that develop metastases are defined by the World Health Organization (WHO) as carcinomas because they cannot be controlled by any available treatments. Pituitary carcinoma is rare, accounting for about $0.1 \%$ of all pituitary adenomas [1]. However, some pituitary adenomas without metastasis display clinically aggressive behavior with multiple recurrences, and they often present a significant therapeutic challenge because conventional therapies may be partially or wholly ineffective. We refer to these tumors as atypical pituitary adenomas (APAs). APAs are

\footnotetext{
* Correspondence: jiaguijun2013@163.com

${ }^{\dagger}$ Equal contributors

'Department of Neurosurgery, Beijing Tiantan Hospital, Capital Medical University, Beijing, China

${ }^{2}$ China National Clinical Research Center for Neurological Diseases (NCRC-ND), 6 Tiantan Xili Street Dongcheng District, Beijing, China
}

thought to be precursor lesions of pituitary carcinoma. Here, we present a case study of an atypical pituitary adenoma with a high degree of malignancy.

\section{Case presentation}

A 46-year-old female presented with one year of frequent recurrent headaches followed by two months of bilateral visual field defects. Her medical history was uneventful. The physical examination was unremarkable. Laboratory tests identified a non-functioning pituitary adenoma. Magnetic resonance imaging revealed an intrasellar and suprasellar tumor. The tumor was partially resected using a transsphenoidal approach in a local hospital in August 2009. Intraoperatively, the tumor appeared to be a typical pituitary adenoma. The postoperative pathology revealed that the tumor was a hormonally inactive benign pituitary adenoma. Her postoperative recovery went well. One week after the operation, she underwent $\gamma$-knife therapy. Her preoperative symptoms had gone into remission. However, one month after the operation, the patient began to feel headaches again, and MRI showed that the tumor had recurred, suggesting extremely rapid regrowth. To cure the disease, she underwent a transcranial operation. The operation outcome was unsatisfactory. Only a 
partial resection was achieved. The preoperative symptoms did not disappear.

She underwent a third surgery in our hospital. Preoperatively, her MRI (see Fig. 1) and CT showed an intrasellar, parasellar and suprasellar mass that had invaded the left cavernous sinus, sphenoid, left orbital bones and left middle cranial fossa. The tumor had become more invasive and much larger. After careful examination, including CT and ultrasonic wave imaging, no metastatic tumors were found at any location in her body. Her symptoms worsened. A transcranial surgery was then performed. An experienced neurosurgeon who had been performing pituitary surgeries for twenty years resected the tumor. Intraoperatively, the tumor was found to be very hard and it bent easily, which was atypical for this type of tumor. The tumor showed marked invasiveness. A near total resection was achieved. A post-operative histological examination revealed that the tumor was an atypical pituitary adenoma with high degrees of malignancy and proliferation. Hematoxylin and eosin staining showed clear cell nucleus heteromorphism and frequent mitosis. In immunocytochemistry, the tumor was divided into a non-function adenoma and a region that was partially positive for vimentin and negative for cytokeratin (CK). Carcinoembryonic antigen (CEA) and epithelial membrane antigen (EMA) staining were also negative. Cluster of differentiation (CD) 35 staining was positive. The tumor was approximately $80 \%$ positive for the proliferative index Kiel University (Ki) 67 (Fig. 2), and it was strongly positive for p53 (Fig. 2).

The patient's postoperative recovery period was unpleasant. Her general health condition worsened each day. Finally, she and her husband decided to stop therapy, and she spent her last days in hospice care. The patient died 7 months after the initial therapy and 1 month after the third operation.

\section{Discussion and conclusions}

Pituitary carcinoma is a very uncommon condition that accounts for merely $0.1 \%$ of all pituitary adenomas. Most pituitary carcinomas develop as a result of invasive relapse or from a previously operated or irradiated invasive adenoma, which may have been the case in this patient. However, this patient did not show any symptoms of metastases. We can only refer to this tumor as an atypical pituitary adenoma, which is defined as a tumor that presents with aggressive biological behavior, an elevated mitotic index (e.g., ki-67 > 3\%) and extensive p53 expression [2]. This patient died 7 months after the first therapy for this tumor was performed. Her prognosis was as bad as for a carcinoma. To some extent, this tumor was more malignant than a pituitary carcinoma, which predicts a poor prognosis with a mean survival of 1.9 years (range, 3 months to 8 years) following the initial diagnosis [3].

Because of the poor prognosis associated with these tumors, when a pituitary atypical adenoma such as this tumor is identified, we should view the tumor differently. In practice, histological, immunohistochemical, radiographic, and ultrastructural analyses are limited in their ability to distinguish between typical and atypical adenomas and malignant carcinomas [1]. A great deal of effort has been made to identify new markers that can be used to distinguish pituitary carcinomas from pituitary tumors with malignant features like the tumor in this patient. Significant advances have been made in the area of the molecular mechanisms that underlie pituitary tumor transformation, and some evidence indicates that transformation from a "benign" adenoma to a pituitary carcinoma is accompanied by accumulative changes in molecular pathway abnormalities. From recently report, we found that cyclin D1 (CCND1), vascular epidermal growth factor (VEGF), Matrix metalloproteinase 9 (MMP-9), microRNAs and Cyclin-dependent kinase inhibitor 1 (p21 $\left.{ }^{\mathrm{Cip} 1}\right)$ were upregulated in pituitary carcinoma, O6-methylguanine-DNA methyltransferase (MGMT), cyclin-dependent kinase inhibitor 2A (P1 $\left.6^{\text {Ink4A }}\right)$, Apoptotic proteins $(\mathrm{Bcl}-2, \mathrm{Bax}, \mathrm{Bcl}-\mathrm{x})$ and Metallothionein isoform 3 (MT3) were downregulated in

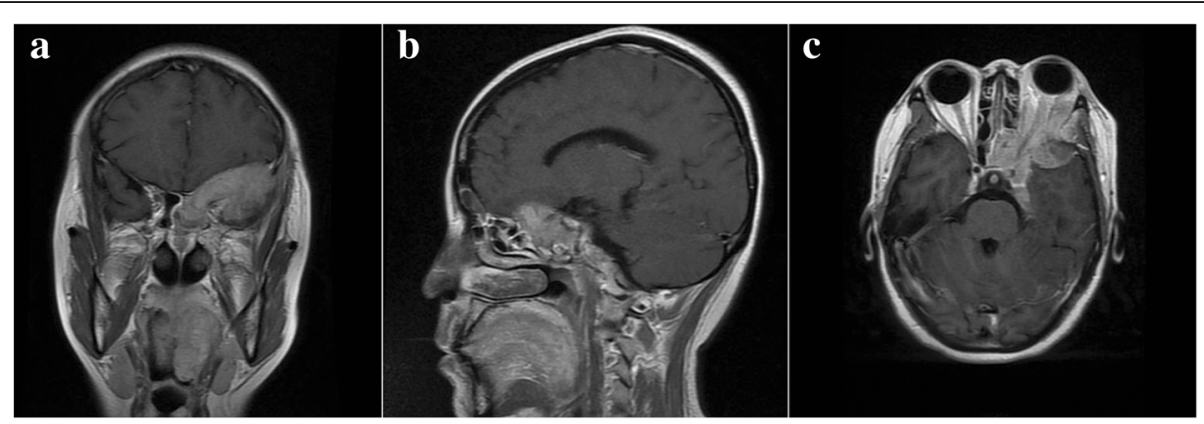

Fig. 1 Magnetic resonance imaging of an atypical pituitary adenoma that consisted of a large mass that had invaded into the left parasellar (a), sphenoid (b), left cavernous sinus and left orbital area (c) 


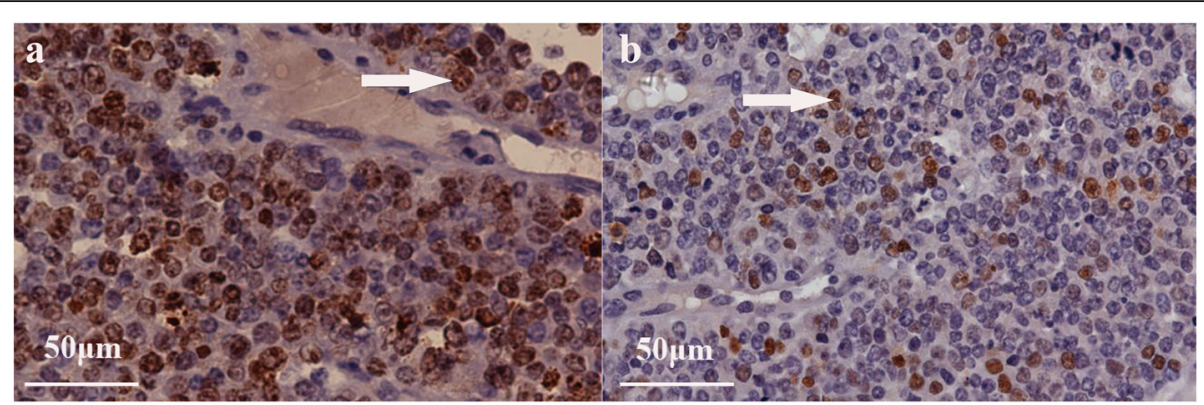

Fig. 2 a Immunohistochemistry showed extremely high ki-67 expression (arrow) (approximately 80\%, X400). b Immunohistochemistry showed strongly positive p53 labeling (arrow) (approximately 15\%, X400)

pituitary carcinoma [4]. However, the details of this process is still unclear.

We did not find any metastases in this patient and therefore could not confirm a diagnosis of carcinoma. But the evidence that were available strongly suggested the malignancy of this tumor. MRI and CT scans showed that the tumor had invaded the surrounding sellar structures, including the cavernous sinus, sphenoid, orbital bones and middle fossa bones. The pathology revealed frequent mitosis, with ki-67 expression in approximately $80 \%$ of the tumor, which was also p53-positive. Some studies have demonstrated that quantitation of the ki-67 labelling index may discriminate between pituitary carcinomas (mean \pm SD for ki-67 LI, $11.9 \pm 3.4 \%$ ) and other adenomas (mean \pm SD for ki-67 LI, $1.4 \pm 0.15 \%$ ) [2]. This finding led to the proposal that pituitary tumors that exhibit a ki-67 labelling index of more than $10 \%$ should be classified as atypical, independent of other criteria [5]. Despite the fact that no prospective studies have supported this conclusion, a high proportion of ki-67-positive cells may remind the clinician to "flag" a patient early in their history because these pituitary tumors exhibit the potential to recur and have the potential to become carcinomas. The patient in this study had a tumor that was approximately $80 \% \mathrm{ki}-67$-positive, which is much greater than a carcinoma and should therefore have been treated in a differently manner than other atypical pituitary adenomas. P53, a tumor suppressor, is a nuclear phosphoprotein that is essential for cell proliferation. While p53 is often found mutated in many human cancers, it is not mutated in pituitary carcinomas. However, nuclear p53 immunoreactivity is correlated with pituitary adenomas invasiveness [5]. Its usefulness in distinguishing atypical pituitary adenoma from pituitary carcinoma is therefore limited. The high proportion of p53-positive cells in the tumor described in this case study was not enough to diagnose it as a carcinoma, but it was enough to indicate that this tumor should have been treated differently.
Pituitary carcinoma is very rare, with one study reporting that only 165 cases have been reported in the English literature. A prospective study involving a large sample size is therefore impossible, and our knowledge of pituitary tumors comes primarily from case reports. We believe that each case of pituitary carcinoma or atypical pituitary adenoma that presents with a high degree of malignancy, such as that described in this case study, should be carefully studied and that this type of clinical experience is as important as the conclusion that come from randomized controlled trials.

In this case study, a high proportion of ki-67 positivity and extensive p53 positivity suggested that this tumor as highly proliferative, and MRI and CT showed that it had invaded into surrounding structures. The patient experienced frequent tumor recurrence, underwent three operations, and finally died within a short period of time. All of these factors support the notion that her tumor was a malignant one. The routine therapy for pituitary carcinoma includes surgery, radiotherapy and chemotherapy, which is the same to treat benign pituitary tumors. However, the outcome from this multimodal treatment regimen was in this case unsatisfactory. Surgical resection did not halt the progression of her tumor, and debulking the tumor size to release her symptoms. Park KS et al. reported that gamma-knife radiosurgery was effective in controlling pituitary carcinoma growth over 3 years [6]. Besides that Novruzov F et al. found that peptide receptor radiotherapy with ${ }^{117} \mathrm{Lu}$ DOTATATE has shown to be effective in stopping pituitary carcinoma growth for more than 4 years [7]. However, there is no clinical trialbased data have demonstrated that traditional radiotherapy is effective in improving survival in patients with pituitary carcinoma [8]. Radiotherapy was also used in this patient, but it did not control the regrowth of the tumor. No randomized prospective studies of systemic chemotherapy have been conducted in patients with pituitary tumors, and the drugs used, in addition to their modes and their durations of administration, have varied widely. This tumor exhibited a high rate of proliferation, 
indicating that it might have been sensitive to chemotherapy. Temozolomide (TMZ), an alkylating chemotherapy drug that was approved for use in glioblastoma therapy, has been used to successfully treat pituitary carcinomas, and it has more recently been used to treat invasive and/or recurrent pituitary adenomas [9]. M Losa et al. reported that temozolomide treatment had a wide range of efficacy in patients with pituitary carcinoma or locally aggressive pituitary adenoma and positive staining for MGMT seems likely to predict a lower chance of response [10]. Our patient did not undergo chemotherapy, but we believe that aggressive chemotherapy might have been a good choice in her case.

In a word, it is not enough to depend solely on the presence of metastasis to diagnose a pituitary carcinoma. Some atypical pituitary adenomas that present with a high rate of proliferation and clear invasion into surrounding structures should be regarded as malignant tumors. Aggressive treatment can be used early in these patients because metastasis can occur in the latest stages of the disease. Moreover, when a clinician encounters an atypical adenoma with such a high ki-67 index, chemotherapy might be a good option, but this needs to be verified in more cases.

\section{Abbreviations}

APAs: Atypical pituitary adenomas; CCND1: Cyclin D1; CD: Cluster of differentiation; CEA: Carcinoembryonic antigen; CK: vimentin and negative for cytokeratin; EMA: Epithelial membrane antigen; MGMT: O6methylguanine-DNA methyltransferase; MMP-9: Matrix metalloproteinase 9; MT3: Metallothionein isoform 3; P16 $6^{\operatorname{lnk} 4 A}$ : cyclin-dependent kinase inhibitor 2A; $21^{\mathrm{Cip} 1}$ : microRNAs and Cyclin-dependent kinase inhibitor 1;

TMZ: Temozolomide; VEGF: Vascular epidermal growth factor

\section{Acknowledgments}

The authors are grateful to Zonggang Hou, Yu Xin and other relevant staff members of Beijing Tiantan Hospital, who helped to perform this study and collect study data. In addition, we would like to thank Junmei Wang for providing pathological determinations.

\section{Funding}

This work was supported by the National Key Technology Research and Development Program of the Ministry of Science and Technology of China (2014BAI04B01) and the Beijing Natural Science Foundation (General Program) (7152050).

\section{Availability of data and materials}

The authors declare that the data supporting the findings of this study are available within the article.

\section{Authors' contributions}

SW and $D L$ reviewed the patient records from hospital, participated in drafted the manuscript. MN participated in the design of the study. GJ conceived of the study, and participated in its design and coordination and helped to draft the manuscript. All authors read and approved the final manuscript.

\section{Ethics approval and consent to participate}

All procedures performed in studies involving human participants were in accordance with the ethical standards of the institutional and/or national research committee and with the 1964 Helsinki declaration and its later amendments or comparable ethical standards. The institutional review board of the Beijing Tiantan hospital approved this study. The reference number of our ethics approval letter was KY2014-021-02.

\section{Consent for publication}

Formal written consent has been obtained from the patient's parents for publication of her clinical information and data. Because the patient has died, we have obtained the consent for publication from her parents.

\section{Competing interests}

The authors declare that they have no competing interests.

Received: 19 December 2016 Accepted: 11 September 2017

Published online: 09 November 2017

\section{References}

1. Heaney AP. Clinical review: Pituitary carcinoma: difficult diagnosis and treatment. J Clin Endocrinol Metab. 2011;96:3649-60.

2. Laws ER Jr, Lopes MB. The new WHO classification of pituitary tumors: highlights and areas of controversy. Acta Neuropathol. 2006;111:80-1.

3. Pernicone PJ, Scheithauer BW, Sebo TJ, Kovacs KT, Horvath E, et al. Pituitary carcinoma: a clinicopathologic study of 15 cases. Cancer. 1997;79:804-12.

4. Yang Z, Zhang T, Gao H. Genetic aspects of pituitary carcinoma: a systematic review. Medicine (Baltimore). 2016;95:e5268.

5. Heaney A. Management of aggressive pituitary adenomas and pituitary carcinomas. J Neuro-Oncol. 2014;117:459-68.

6. Park KS, Hwang JH, Hwang SK, Kim S, Park SH. Pituitary carcinoma with fourth ventricle metastasis: treatment by excision and gamma-knife radiosurgery. Pituitary. 2014;17:514-8.

7. Novruzov F, Aliyev JA, Jaunmuktane Z, Bomanji JB, Kayani I. The use of (68)Ga DOTATATE PET/CT for diagnostic assessment and monitoring of (177)Lu DOTATATE therapy in pituitary carcinoma. Clin Nucl Med. 2015;40:47-9.

8. Hansen TM, Batra S, Lim M, Gallia GL, Burger PC, et al. Invasive adenoma and pituitary carcinoma: a SEER database analysis. Neurosurg Rev. 2014;37: 279-85.

9. Liu JK, Patel J, Eloy JA. The role of temozolomide in the treatment of aggressive pituitary tumors. J Clin Neurosci. 2015;22:923-9.

10. Losa M, Mazza E, Terreni MR, McCormack A, Gill AJ, et al. Salvage therapy with temozolomide in patients with aggressive or metastatic pituitary adenomas: experience in six cases. Eur J Endocrinol. 2010;163:843-51.

Submit your next manuscript to BioMed Central and we will help you at every step:

- We accept pre-submission inquiries

- Our selector tool helps you to find the most relevant journal

- We provide round the clock customer support

- Convenient online submission

- Thorough peer review

- Inclusion in PubMed and all major indexing services

- Maximum visibility for your research

Submit your manuscript at www.biomedcentral.com/submit
C Biomed Central 